\title{
Análisis de la eficiencia de un amortiguador combinado sintonizado con incertidumbre en los parámetros sometido a excitaciones sísmicas de bajo contenido de frecuencias
}

\section{Analysis of the efficiency of a combined tuned damper with uncertainty in the parameters} subject to seismic excitations of low frequency content

Fecha de entrega: 24 de junio 2020 Fecha de aceptación: 29 de octubre 2020

\section{Gilda Espinoza, Sebastián Rivas y Álvaro Suazo}

Departamento de Ingeniería Civil y Ambiental, Universidad del Bío-Bío, Avenida Collao 1202, Casilla 5-C, Concepción, Chile, gespinoz@ubiobio.cl,searivas@alumnos.ubiobio.cl,asuazo@ubiobio.cl

Este estudio analiza la eficiencia en la reducción del desplazamiento lateral de una estructura bajo excitaciones sísmicas estocásticas de bajo contenido de frecuencias. El dispositivo de estudio es el Amortiguador Combinado Sintonizado (ACS). El objetivo es analizar cómo cambia su eficiencia, cuando se introduce incertidumbre en sus parámetros de diseño. El ACS está compuesto por dos dispositivos efecto-masa, un amortiguador de masa sintonizado (AMS) y un amortiguador de columna de líquido sintonizado (ACLS). Para trabajar con la componente no lineal de la ecuación del ACLS en el análisis estocástico, se utilizó la técnica de linealización estadística equivalente. Se considera una incertidumbre de \pm 5 y $\pm 10 \%$. Los parámetros a los que se les introdujo incertidumbre, fueron la razón de longitud del ACLS y la razón de masa. Como criterio de optimización, se consideró la reducción de la desviación estándar del desplazamiento del sistema principal. Los resultados muestran que la eficiencia del ACS es máxima, cuando el periodo de la estructura, coincide con el periodo predominante de la excitación sísmica. Por otra parte, existen leves variaciones en la eficiencia del ACS, cuando se introduce una incertidumbre de hasta un $10 \%$ en la razón de masa o razón de longitud del ACLS.

Palabras clave: ACS, ACLS, AMS, análisis estocástico, incertidumbre en los parámetros, bajo contenido de frecuencias
This study analyses the efficiency, in reducing the lateral displacement of a structure, under stochastic seismic excitations of low frequency content. The study device is the Tuned Combined Damper (TCD). The objective is to analyze how its efficiency changes when uncertainty is introduced in the design parameters. The TCD is composed of two mass-effect devices, a tuned mass damper (TMD) and a tuned liquid column damper (TLCD). To work with the nonlinear component of the TLCD equation in stochastic analysis, the equivalent statistical linearization technique was used. Uncertainties of \pm 5 and $\pm 10 \%$ are considered. The parameters to which uncertainty was introduced were the length ratio of the TLCD and the mass ratio. As an optimization criterion, the reduction of the standard deviation of the displacement of the main system was considered. The results show that the efficiency of the TCD is maximum when the period of the structure coincides with the predominant period of seismic excitation. On the other hand, there are slight variations in the efficiency of the TCD when an uncertainty of up to $10 \%$ is introduced in the mass or length ratio of the TLCD.

Keywords: CTD, TLCD, TMD, stochastic analysis, parameter uncertainty, low frequency content

\section{Introducción}

A medida que la civilización avanza, el aumento de población de las ciudades, ha implicado un diseño de estructuras más altas y esbeltas. Esto ha implicado nuevos desafíos en el control de estas estructuras, cada vez más flexibles, ante cargas dinámicas como viento y sismo. Como una alternativa al diseño tradicional, se ha desarrollado el diseño por reducción de vibraciones, llamado también de protección sísmica. Los sistemas de protección sísmicos son los sistemas pasivos, porque no necesitan de una fuente de energía externa. Entre estos se encuentran los dispositivos de efecto-masa. Los más utilizados son el Amortiguador de 
Masa Sintonizado (AMS) y el Amortiguador de Columna de Líquido Sintonizado (ACLS).

El AMS consiste en una masa unida a la estructura a través de un amortiguador viscoso y un resorte elástico lineal. Generalmente, se ubican en el último piso o azotea del edificio (Schmelzer et al., 2010; Tributsch y Adam, 2012; Bigdeli y Kim, 2015)

El objetivo del AMS es sintonizar su frecuencia con la frecuencia fundamental de la estructura principal para provocar resonancia entre ellos, entonces la energía sísmica recibida por el edificio es disipada por la fuerza inercial del AMS que reacciona sobre éste en el sentido horizontal. Por otra parte, el ACLS es un tubo en U que posee en su interior líquido, generalmente agua, que ante un movimiento en su base, oscila con una frecuencia propia. En su parte inferior, posee un orificio que provoca una pérdida de carga (Shum, 2009). Di Matteo et al. (2015), conociendo que la ecuación que gobierna el comportamiento del ACLS es de naturaleza no lineal, calibran los parámetros del ACLS para una fase de pre-diseño. Y validan la calibración a través de un análisis experimental de un edificio de corte, con una excitación de ruido blanco.

Espinoza et al. (2018a) estudian la eficiencia de un ACLS en el control de estructuras con comportamiento no lineal. Encuentran que el ACLS se sintoniza con la frecuencia lineal equivalente, cuando la excitación es de ancho de banda amplio, y se sintoniza con la frecuencia predominante del input cuando el ancho de banda es angosto. Wang et al. (2016) proponen un Amortiguador Combinado Sintonizado (ACS), que combina un AMS con un ACLS. Este estudio fue realizado para edificios de gran altura que se comportan dentro del rango lineal y que son sometidos a una excitación de viento. Di Matteo et al. (2017) realizan un estudio analítico del ACS que posteriormente es validado experimentalmente. Chen y Yang (2018) estudian experimentalmente la reducción de desplazamiento de una estructura lineal AMS y ACLS y diferentes razones de masa del AMS y distintas razones de altura del líquido del ACLS para determinar un AMSACLS híbrido. Debbarma et al. (2010) observan que si los parámetros no están perfectamente sintonizados en el modo de vibración que se quiere controlar, la eficiencia de los amortiguadores puede reducirse. Esto tiene una gran implicancia puesto que se debiera entonces considerar la presencia de incertidumbre en los parámetros del sistema. Por lo tanto, prácticamente no existen investigaciones que incorporen el efecto de la incertidumbre en el análisis de un ACS. Si bien Debbarma et al. (2010) realizan una investigación sobre el comportamiento de los parámetros óptimos de un amortiguador de columna de líquido sintonizado ACLS, cuando incluyen incertidumbre en los parámetros no caracterizan la excitación sísmica, ni analizan la eficiencia.

Por ello, esta investigación tiene por objetivo incorporar la incertidumbre en el sistema para analizar el comportamiento de los parámetros óptimos del ACS y su eficiencia en el control de una estructura sometida a una excitación sísmica estocástica de bajo contenido de frecuencias. El caso de una excitación sísmica de alto contenido de frecuencias con incertidumbre es abordado por Espinoza et al. (2020).

\section{Modelo estructural}

La Figura 1 muestra un sistema de Amortiguador Combinado Sintonizado ACS, correspondiente a la unión de un AMS y un ACLS en paralelo, el cual está unido a una estructura con comportamiento lineal cuyos parámetros $M_{s}, C_{s}$ y $k_{s}$ corresponden a la masa, amortiguamiento y rigidez del sistema principal. De igual manera, $M_{T}, C_{T} \mathrm{y}$ $k_{T}$ corresponden a la masa, amortiguamiento y rigidez del AMS, $B_{h}$ y $h$ son las dimensiones horizontal y vertical del sistema ACLS y $\ddot{u}_{g}$ es la aceleración del suelo.

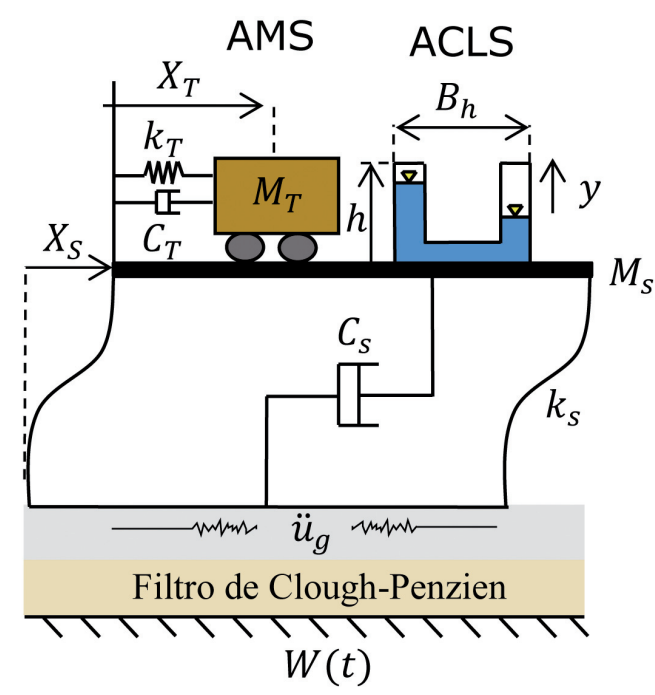

Figura 1: Estructura con ACS. Modelo1 (M1)

El sistema está sometido a un input estocástico que se determina a partir de la generación de una señal de ruido blanco en la roca, que pasa a través de un estrato de suelo 
a la base de la estructura, generando una aceleración basal $\ddot{u}_{g}$. Este estrato de suelo se modela, a través del filtro de Clough-Penzien (Clough y Penzien, 1975), compuesto por dos filtros, el primero emula el estrato de suelo y un segundo filtro que corrige las frecuencias bajas, es decir, no las elimina completamente. Las frecuencias bajas están asociadas a periodos mayores a $4 \mathrm{~s}$. Los parámetros característicos de estos filtros, dan la característica de una excitación de ancho de banda angosto NBP (narrow band process) o bajo contenido de frecuencias. El periodo predominante del filtro es de $2 \mathrm{~s}$, lo cual representa el estrato del suelo sobre la roca que no interactúa con la estructura. El caso de interacción suelo- estructura puede ser revisado en Espinoza et al. (2018b). Los grados de libertad del sistema están definidos por el vector:

$$
\mathbf{X}=\left\{X_{S}(t) \quad X_{T}(t) \quad y(t)\right\}^{T}
$$

donde $X_{s}$ representa el desplazamiento horizontal de la estructura principal, $X_{T}$ representa el desplazamiento lateral del AMS e $y$ indica el desplazamiento de la superficie del líquido dentro del ACLS en sentido vertical.

\section{Linealización estadística equivalente LEE}

La LEE permite reemplazar las ecuaciones no lineales por ecuaciones lineales a través de técnicas de linealización estadística. En este caso, se linealiza la ecuación no lineal del ACLS, que corresponde a una ecuación diferencial de segundo orden con un amortiguador de líquido acoplado a la estructura que es excitado basalmente a través de una aceleración $\ddot{u}_{g}$ (Sakai et al., 1989).

$$
\rho A L_{e} \ddot{y}+2 \rho A \xi_{l}\lfloor\dot{y}\rfloor \dot{y}+2 \rho g A y=-\rho A B_{h}\left(\ddot{x}+\ddot{u}_{g}\right)
$$

Para la linealización estadística equivalente, se reemplaza el término no lineal por un amortiguamiento lineal equivalente.

$$
C_{P}=\frac{\xi_{l} \sigma_{\dot{y}}}{\sqrt{2 \pi}}
$$

Donde $\sigma_{\dot{y}}$ es la desviación estándar de la velocidad del líquido y $\xi_{l}$ es el coeficiente de pérdida de carga. Finalmente, la ecuación del ACLS linealizada queda de la siguiente manera:

$$
\rho A L_{e} \ddot{y}+2 \rho A C_{P} \dot{y}+2 \rho g A y=-\rho A B_{h}\left(\ddot{x}+\ddot{u}_{g}\right)
$$

\section{Ecuación lineal de movimiento del ACS}

Las ecuaciones del movimiento del modelo M1 sometido a excitaciones sísmicas estocásticas, se obtuvieron de las ecuaciones de Euler-Lagrange y son presentadas a continuación:

$$
\begin{aligned}
& M_{T}\left(\ddot{X}_{T}+\ddot{X}_{S}+\ddot{u}_{g}\right)+C_{T} \dot{X}_{T}+K_{T} X_{T}=0 \\
& 2 M_{V L} \ddot{y}+M_{H L}\left(\ddot{y}_{T}+\ddot{X}_{S}+\ddot{u}_{g}\right)+K_{L} \dot{y}+C_{L} y=0 \\
& \left(M_{S}+M_{T}+2 M_{v l}+M_{h l}\right) \ddot{X}_{S}+M_{T} \ddot{X}_{T}+ \\
& \left(M_{S}+M_{T}+2 M_{v l}+M_{h l}\right) \ddot{u}_{g}+M_{h l} \ddot{y}+C_{s} \dot{x}_{s}+k_{s} x_{s}=0
\end{aligned}
$$

Siendo $\ddot{X}_{s}$ la aceleración de la estructura principal, $\ddot{X}_{T}$ y $\dot{X}_{T}$, la aceleración y la velocidad del AMS respectivamente y $\ddot{u}_{g}$ es la aceleración basal. Además $\mu_{T}$ es la razón de masa del AMS respecto de la estructura principal, $\xi_{T}$ y $\omega_{T}$ son la razón de amortiguamiento y la frecuencia del amortiguador respectivamente. $M_{V L}$ y $M_{H L}$ son la masa del líquido vertical y horizontal respectivamente, $\ddot{y}_{T}$ es la aceleración del líquido e $\dot{y}_{T}$ es la velocidad del líquido. A raíz de la ecuación (9), se definen los parámetros del ACLS. Los parámetros del AMS son:

$$
\mu_{T}=\frac{M_{T}}{M_{S}} \quad ; \quad C_{T}=2 \xi_{T} M_{T} \omega_{T} \quad ; \quad K_{T}=\omega_{T}^{2} \mu_{T} M_{S}
$$

Y los parámetros del ACLS son:

$$
\begin{array}{ll}
M_{L}=\rho A L_{e} & ; \quad M_{L H}=\rho A B_{h} \quad ; \\
C_{L}=2 \rho A C_{P} & ; \quad K_{L}=2 \rho g A
\end{array}
$$

donde $\rho$ es la densidad del líquido, $A$ es el área de la sección transversal del tubo, $L_{e}$ es la longitud efectiva de la columna liquida y $g$ es la aceleración de gravedad.

Se definen los parámetros de la estructura como:

$$
C_{S}=2 \xi_{S} M_{S} \omega_{S} \quad ; \quad K_{S}=\omega_{S}^{2} M_{S}
$$

Donde $\omega_{s}$ es la frecuencia de la estructura principal y $\xi_{s}$ es la razón de amortiguamiento de la estructura principal.

\section{Representación en formato de estado}

Las ecuaciones planteadas pueden representarse matricialmente al definir igualdades matemáticas entre ellas, entonces se normalizan respecto a la masa de la 
estructura principal las diferentes masas, amortiguamientos y rigideces del sistema combinado. A partir de las ecuaciones (8) a (10) se definen las siguientes expresiones:

$$
\begin{aligned}
& \frac{M_{T}}{M_{S}}=\mu_{T} \quad ; \quad \frac{C_{T}}{M_{S}}=\frac{2 \xi_{T} M_{T} \omega_{T}}{M_{S}}=2 \xi_{T} \mu_{T} \omega_{T} \quad ; \\
& \frac{K_{T}}{M_{T}}=\frac{\omega_{T}^{2} \mu_{T} M_{S}}{M_{S}}=\omega_{T}^{2} \mu_{T}
\end{aligned}
$$

En el ACLS se define $\mu_{\mathrm{L}}$ como la razón de masa entre el amortiguador del líquido y la estructura principal y $p$ como la razón de longitud,

$$
\begin{aligned}
& \mu_{L}=\frac{M_{L}}{M_{S}} ; \quad p=\frac{B_{h}}{L_{e}} \\
& \frac{M_{L h}}{M_{S}}=\frac{\rho A B_{h}}{M_{S}}=\frac{\rho A L_{e} p}{M_{S}}=\frac{M_{L} p}{M_{S}}=\mu_{L} p \\
& \frac{C_{L}}{M_{S}}=\frac{2 \rho A C_{P}}{M_{S}}=\frac{2 \rho A C_{P} \mu_{L}}{M_{L}}=\frac{2 \rho A C_{P} \mu_{L}}{\rho A L_{e}}=\frac{2 C_{P} \mu_{L}}{L_{e}} \\
& \frac{K_{L}}{M_{S}}=\frac{2 \rho g A}{M_{S}}=\frac{2 \rho g A \mu_{L}}{\rho A L_{e}}=\frac{2 g \mu_{L}}{L_{e}}
\end{aligned}
$$

Usando (5) a (7) y ocupando (11) a (13), se obtienen las siguientes matrices:

$$
\begin{aligned}
& \mathbf{M}=\left[\begin{array}{ccc}
1+\mu_{T}+\mu_{L} & \mu_{T} & \rho \mu_{L} \\
\mu_{T} & \mu_{T} & 0 \\
p \mu_{L} & 0 & \mu_{L}
\end{array}\right] ; \\
& \mathbf{C}=\left[\begin{array}{ccc}
2 \xi_{S} \omega_{S} & 0 & 0 \\
0 & 2 \xi_{T} \omega_{T} \mu_{T} & 0 \\
0 & 0 & 2 \frac{C_{P} \mu_{L}}{L e}
\end{array}\right] \\
& \mathbf{K}=\left[\begin{array}{ccc}
\omega_{S}^{2} & 0 & 0 \\
0 & \mu_{T} \omega_{T}^{2} & 0 \\
0 & 0 & \frac{2 g \mu_{L}}{L e}
\end{array}\right] \quad ; \quad \mathbf{B}_{\mathbf{u}}=\left[\begin{array}{c}
-\left(1+\mu_{T}+\mu_{L}\right) \\
-\mu_{T} \\
-\mu_{L} p
\end{array}\right]
\end{aligned}
$$

\section{Filtro de Clough-Penzien}

En este estudio el movimiento del suelo es modelado a través de un filtro de Clough-Penzien, que genera el input sísmico como un proceso estocástico estacionario de ancho de banda angosto NBP (narrow broadband process). Este input se genera a partir de un ruido blanco. $S_{g}(\omega)$ es la matriz de Densidad de Potencia Espectral PSD (Power Spectral Density) del movimiento horizontal del suelo, la cual se expresa de la siguiente forma:

$$
S_{g}(\omega)=S(\omega) I
$$

Siendo $S(\omega)$ la función PSD del movimiento del suelo que representa el NBP a partir del filtro:

$$
\begin{aligned}
& S_{g}(\omega)=S_{o} \frac{\omega_{g}+4 \xi_{g}^{2} \omega_{g}^{2} \omega^{2}}{\left(\omega_{g}^{2}-\omega^{2}\right)^{2}+4 \xi_{g}^{2} \omega_{g}^{2} \omega^{2}} . \\
& \frac{\omega^{4}}{\left(\omega_{f}^{2}-\omega^{2}\right)^{2}+4 \xi_{f}^{2} \omega_{f}^{2} \omega^{2}}
\end{aligned}
$$

donde $S_{o}, \omega_{g}, \xi_{g}, \omega_{f}, \mathrm{y} \xi_{f}$ representan la intensidad de ruido blanco, frecuencia del estrato de suelo, razón de amortiguamiento del estrato de suelo, frecuencia del filtro y razón de amortiguamiento del filtro respectivamente. Los parámetros del filtro se obtuvieron mediante un ajuste por mínimos cuadrados del PSD del sismo de México en 1985, el cual es un sismo de bajo contenido de frecuencias, con un periodo predominante de $2 \mathrm{~s}$. El valor de los parámetros, se encuentran en la Tabla 1.

Tabla 1: Parámetros del filtro de Clough - Penzien

\begin{tabular}{|c|c|c|c|c|c|}
\hline $\begin{array}{c}\text { Tipo de } \\
\text { entrada }\end{array}$ & $S_{o}$ & $\omega_{g}, \mathrm{rad} / \mathrm{s}$ & $\xi_{g}$ & $\omega_{f}, \mathrm{rad} / \mathrm{s}$ & $\xi_{f}$ \\
\hline NBP & 207.23 & 3.14 & 0.1 & 8.48 & 0.9 \\
\hline
\end{tabular}

Se define del vector de estado del filtro como (Saitua et al., 2018):

$\mathbf{X}_{\mathbf{f}}=\left\{\begin{array}{llll}X_{g} & \dot{X}_{g} & X_{f} & \dot{X}_{f}\end{array}\right\}^{T}$

donde $X_{g}, \dot{X}_{g}$ representan el desplazamiento y velocidad del suelo, mientras que $X_{f}$ y $\dot{X}_{f}$ son el desplazamiento y velocidad del filtro. Las ecuaciones de estado se definen como sigue:

$$
\dot{\mathbf{X}}_{\mathbf{f}}=\mathbf{A}_{\mathbf{f}} \mathbf{X}_{\mathbf{f}}+\mathbf{B}_{\mathbf{f}} W(t) \quad ; \quad \ddot{u}_{g}=\mathbf{C}_{\mathbf{f}} \mathbf{X}_{\mathbf{f}}
$$

donde las matrices $\mathbf{A}_{\mathrm{f}}, \mathbf{B}_{\mathrm{f}}$ y $\mathbf{C}_{\mathrm{f}}$ corresponden a la matriz de estado del filtro, la matriz de colocación del filtro y la matriz del output del filtro.

$$
\begin{aligned}
& \mathbf{A}_{\mathbf{f}}=\left[\begin{array}{cccc}
0 & 1 & 0 & 0 \\
-\omega_{g}^{2} & -2 \xi_{g} \omega_{g} & 0 & 0 \\
0 & 0 & 0 & 1 \\
\omega_{g}^{2} & 2 \xi_{g} \omega_{g} & -\omega_{f}^{2} & -2 \xi_{f} \omega_{f}
\end{array}\right] ; \quad \mathbf{B}_{\mathbf{f}}=\left[\begin{array}{c}
0 \\
-1 \\
0 \\
0
\end{array}\right] \\
& \mathbf{C}_{\mathbf{f}}=\left\{\begin{array}{llll}
\omega_{g}^{2} & 2 \xi_{g} \omega_{g} & -\omega_{f}^{2} & -\xi_{f} \omega_{f}
\end{array}\right\}
\end{aligned}
$$

Se extiende el estado para obtener la matriz aumentada del sistema combinado. 
Considerando el vector de estado extendido como:

$$
\begin{aligned}
& \mathbf{X}_{2}=\left\{\begin{array}{lllllll}
X_{S}(t) & X_{T}(t) & y(t) & \dot{X}(t) & \dot{X}_{S}(t) & \dot{y}(t) & X_{g}(t) \\
\dot{X}_{g}(t) & X_{f}(t) & \left.\dot{X}_{f}(t)\right\}^{T} & & &
\end{array}\right.
\end{aligned}
$$

Entonces la ecuación de estado es igual a:

$$
\dot{\mathbf{X}}_{2}=\mathbf{A}_{2} \mathbf{X}_{2}+\mathbf{B}_{2} W(t)
$$

y las matrices de estado del estado extendido son:

$$
\begin{aligned}
& \mathbf{A}_{2}=\left[\begin{array}{cc}
\mathbf{A} & \mathbf{B C}_{\mathrm{f}} \\
0 & \mathbf{A}_{\mathrm{f}}
\end{array}\right] \\
& \mathbf{B}_{2}=\left[\begin{array}{c}
0 \\
\mathbf{B}_{\mathrm{f}}
\end{array}\right]
\end{aligned}
$$

donde $\mathbf{A}_{2}$ y $\mathbf{B}_{2}$ corresponde a la matriz y al vector de excitación del sistema combinado (ACLS + estructura + filtro), mientras que $\mathbf{A}_{\mathrm{f}}$ y $\mathbf{B}_{\mathrm{f}}$ son la matriz y vector de excitación del filtro (suelo + filtro).

\section{Cálculo de matriz de covarianza}

La solución estacionaria se obtuvo resolviendo la ecuación matricial (25) de Lyapunov (1992), para un estado estacionario y considerando ruido blanco como entrada. Como resultado se obtuvo así la matriz de covarianza de respuesta $\mathbf{R}$ que contiene las varianzas, desviaciones estándar y coeficientes de correlación de los grados de libertad del sistema extendido. Para el ruido blanco, se consideró una varianza de la aceleración del suelo en la entrada del sistema combinado.

$$
\sigma_{\ddot{u}_{g}}^{2}=\frac{\mathrm{PGA}}{3}
$$

Donde PGA es el peak ground acceleration considerado, correspondiente a $0.3 \mathrm{~g}$ para este estudio, de modo que el sistema se asume dentro del rango lineal. Para verificar este supuesto se debe plantear un modelo histerético donde sea posible evaluar si la rigidez se degrada o no (Espinoza et al., 2018a). Se presenta a continuación la ecuación de Lyapunov:

$$
\mathbf{A}_{\mathbf{2}} \mathbf{R}+\mathbf{R} \mathbf{A}_{2}^{\mathrm{T}}+\mathbf{B}_{2} W \mathbf{B}_{2}^{\mathrm{T}}=0
$$

donde $\mathbf{R}$ corresponde a la matriz de covarianza del sistema completo y $W$ a la intensidad del ruido blanco.

\section{Inclusión de incertidumbre en $\mu$ y $p$}

Dado que las matrices ampliadas del sistema combinado $\mathbf{A}_{2}$ y $\mathbf{B}_{2}$ involucran parámetros del sistema inciertos, pero limitados, la solución asociada a la matriz de covarianza de respuesta $\mathbf{R}$ también los involucrará. Es por ello que, para incluir esta incertidumbre en los parámetros y en el cálculo de la matriz de covarianza, se aproximaron las matrices del sistema $\mathbf{A}_{2}, \mathbf{B}_{2}$ y $\mathbf{R}$ en series de Taylor de primer orden como media y parte fluctuante como se muestra a continuación:

$$
\begin{aligned}
& \left(\overline{\mathbf{A}}_{2}+\sum_{i=1}^{m} \frac{\partial \mathbf{A}_{2}}{\partial z_{i}} \delta z_{i}+\cdots\right)\left(\overline{\mathbf{R}}+\sum_{i=1}^{m} \frac{\partial \mathbf{R}}{\partial z_{i}} \delta z_{i}+\cdots\right)+ \\
& \left(\overline{\mathbf{R}}+\sum_{i=1}^{m} \frac{\partial \mathbf{R}}{\partial z_{i}} \delta z_{i}+\cdots\right)\left(\overline{\mathbf{A}}_{2}+\sum_{i=1}^{m} \frac{\partial \mathbf{A}_{2}}{\partial z_{i}} \delta z_{i}+\cdots\right)+
\end{aligned}
$$$$
\left(\overline{\mathbf{B}}_{2}+\sum_{i=1}^{m} \frac{\partial \mathbf{B}_{2}}{\partial z_{i}} \delta z_{i}+\cdots\right)=0
$$

Donde $\overline{\mathbf{A}}_{2}, \overline{\mathbf{B}}_{2}$ y $\overline{\mathbf{R}}$, y representan la matriz correspondiente al valor nominal del parámetro incierto, pero limitado. Dejando de lado los términos de orden superior y las ecuaciones del término de orden igual, para los parámetros inciertos pero limitados, se puede obtener lo siguiente (Debbarma et al., 2010):

$$
\begin{aligned}
& \overline{\mathbf{A}}_{\mathbf{2}} \overline{\mathbf{R}}+\overline{\mathbf{R}} \overline{\mathbf{A}}_{2}^{\mathrm{T}}+\overline{\mathbf{B}}_{\mathbf{2}} W=0 \\
& \overline{\mathbf{A}}_{\mathbf{2}} \frac{\partial \mathbf{R}}{\partial z_{i}}+\frac{\partial \mathbf{A}_{\mathbf{2}}}{\partial z_{i}} \overline{\mathbf{R}}+\overline{\mathbf{R}} \frac{\partial \mathbf{A}_{\mathbf{2}}{ }^{\mathrm{T}}}{\partial z_{i}}+\frac{\partial \mathbf{R}}{\partial z_{i}} \overline{\mathbf{A}}_{2}{ }^{\mathrm{T}}+\overline{\mathbf{B}}_{2} W=0
\end{aligned}
$$

donde se obtiene la matriz de covarianza media $\mathbf{R}$ y la matriz de sensibilidad de primer orden de la matriz de covarianza $\frac{\partial \mathbf{R}}{\partial z_{i}}$ de (28), siendo $W$ la intensidad del ruido blanco. Para obtener los valores de las desviaciones estándar de los desplazamientos con incertidumbre positiva y negativa. A continuación se muestra la desviación estándar del desplazamiento de la estructura, en desarrollo de serie de Taylor.

$$
\begin{gathered}
\sigma_{x_{s}}=\bar{\sigma}_{x_{s}} \pm \sum_{i=1}^{m} \frac{\partial \sigma_{x_{s}}}{\partial z_{i}} \delta z_{i} \pm \cdots \\
\left\{\begin{array}{l}
\sigma_{x_{s_{\text {sup }}}}=\bar{\sigma}_{x_{s}}+\sum_{i=1}^{m} \frac{\partial \sigma_{x_{s}}}{\partial z_{i}} \delta z_{i} \pm \cdots \\
\sigma_{x_{\text {slow }}}=\bar{\sigma}_{x_{s}}-\sum_{i=1}^{m} \frac{\partial \sigma_{x_{s}}}{\partial z_{i}} \delta z_{i} \pm \cdots
\end{array}\right.
\end{gathered}
$$

donde:

$$
\sigma_{x_{S}}=\sqrt{\mathbf{R}(1,1)} \quad ; \quad \frac{\partial \sigma_{x_{S}}}{\partial z_{i}}=\frac{1}{2}\left[\frac{\frac{\partial \mathbf{R}(1,1)}{\partial z}}{\sqrt{\mathbf{R}(1,1)}}\right]
$$


El nivel de incertidumbre considerado es de $\pm 5 \mathrm{y} \pm 10 \%$, donde es la variable en la que se incluye la incertidumbre correspondiendo en esta investigación a la razón de masa $\mu$ y la razón de longitud $p$.

\section{Procedimiento de optimización}

Se propone $J$ como función objetivo para minimizar la desviación estándar del desplazamiento de la estructura principal.

Criterio:

$$
\begin{aligned}
& J\left(\gamma_{l}, \xi_{R}, \gamma_{T}, \xi_{T}\right)=\min \left(\sigma_{x_{S}}\right) \\
& J\left(\gamma_{l}, \xi_{R}, \gamma_{T}, \xi_{T}\right)=\min \left(\sigma_{x_{S} \text { up }}\right) \\
& J\left(\gamma_{l}, \xi_{R}, \gamma_{T}, \xi_{T}\right)=\min \left(\sigma_{x_{S} \text { low }}\right)
\end{aligned}
$$

Sujeto a:

$$
\begin{array}{ll}
0.5<\gamma_{T}<2 & ; \quad 0<\xi_{T}<1 \\
0.5<\gamma_{L}<2 & ; \quad 0<\xi_{L}<30 \\
h-c \sigma_{y} \geq 0 &
\end{array}
$$

donde $c$ es el peak factor que en esta investigación asume un valor igual a 2.5, y corresponde al límite máximo de desplazamiento en altura que puede alcanzar el líquido dentro del ACLS; $\gamma_{T}$ y $\gamma_{L}$ son las razones de sintonía del AMS y ACLS, respectivamente, que se muestran a continuación:

$$
\gamma_{T}=\frac{\omega_{T}}{\omega_{S}} \quad ; \quad \gamma_{L}=\frac{\omega_{L}}{\omega_{S}}
$$

\section{Análisis y resultados}

A continuación, se presenta el análisis de resultados para el ACLS y del AMS, que son los dispositivos que componen el ACS. El análisis se realiza considerando una variación de la razón de masa $\mu$ y de la razón de longitud $p$ del ASC. Como ya se ha mencionado, se consideró una incertidumbre de $\pm 5 \mathrm{y} \pm 10 \%$, respecto al valor $\sin$ incertidumbre. Los resultados del análisis se presentan en figuras compuestas por 2 filas y 3 columnas de gráficos cada una. Las filas representan a los parámetros óptimos de diseño del ASC compuesto por el ACLS y AMS. Mientras tanto las columnas definen la variación del periodo de la estructura principal para $1.5,2.0$ y 2.5 s. Además, cada gráfico presenta una curva sin incertidumbre (línea azul), y las respectivas variaciones correspondientes a la incertidumbre de un 5\% (línea segmentada roja), $-5 \%$ (línea continua roja), 10\% (línea segmentada negra) y $-10 \%$ (línea continua negra).

\section{Análisis del comportamiento de un ACLS con variación de $\mu$}

La Figura 3 muestra los parámetros óptimos del ACLS, en función de la razón de masa $\mu$, considerada como un $50 \%$ de la razón de masa del ACLS más un 50\% de la razón de masa del AMS. Se observa que la razón de frecuencia óptima del ACLS disminuye en forma cuadrática respecto a $\mu$, para un periodo de $1.5 \mathrm{~s}$ y aumenta respecto a la flexibilidad de la estructura, con un decrecimiento en forma lineal. En cuanto a la incertidumbre se observa una mayor variación de la razón de frecuencias para una estructura rígida. En estructuras más flexibles este parámetro es insensible a la incertidumbre en $\mu$. En cuanto al coeficiente de pérdida de carga, existe un decrecimiento del coeficiente de pérdida de carga para estructuras rígidas e intermedias y un aumento para estructuras más flexibles, y es insensible a una incertidumbre en $\mu$.
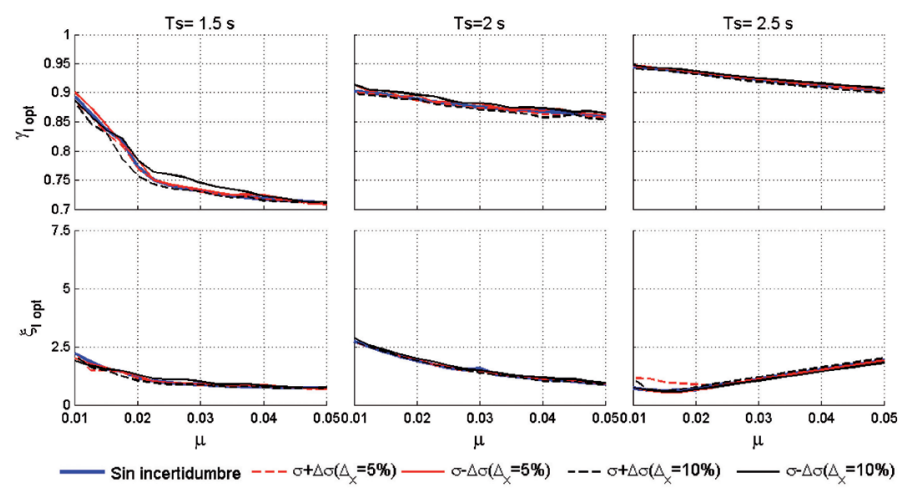

Figura 2: Parámetros óptimos del ACLS en función de la razón de masa $\mu$

\section{Análisis del comportamiento de un AMS con variación de $\mu$}

Con respecto a los parámetros óptimos del AMS, se observa en la Figura 3 que en el caso de estructura más rígida de $1.5 \mathrm{~s}$, la razón de frecuencia disminuye con el aumento de razón de masa. Las estructuras de periodo intermedio prácticamente son insensibles a la variación de la razón de masa, y para estructuras de periodo alto, la 
razón de frecuencia del ACLS aumenta con respecto a la razón de masa. Por otra parte, se observa que cuando las estructuras son más rígidas y más flexibles, el parámetro óptimo de la razón de frecuencia de líquido, tiene una mayor incertidumbre hacia razón de masa mayores. Con respecto a la razón de amortiguamiento, se observa que, para los 3 periodos analizados, aumenta con la razón de masa. En cuanto a la incertidumbre en la razón de masa, se produce que, para periodos rígidos y flexibles, tienen una mayor influencia en los óptimos del AMS.

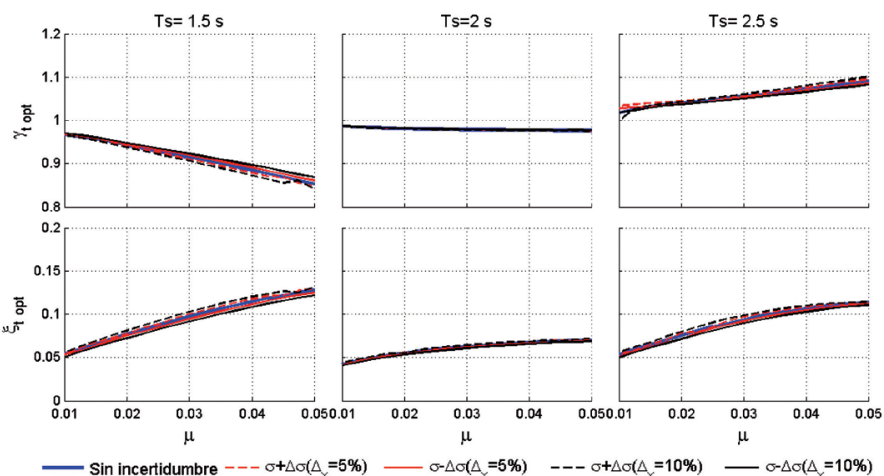

Figura 3: Parámetros óptimos del AMS en función de la razón de masa $\mu$

\section{Análisis del comportamiento de un ACLS con variación de $p$}

La Figura 4 muestra el comportamiento de los parámetros óptimos cuando se introduce una incertidumbre en la razón de longitud $p$ del ACLS, la distribución de los gráficos y valores asociados de los periodos de la estructura, por columnas, son los mismos de la Figura 2. Se observa que, en el caso de estructuras rígidas a intermedias, hay una disminución de la razón de sintonía óptima, y para estructuras flexibles es prácticamente constante, a medida que aumenta $p$. También se puede observar, que la incertidumbre es prácticamente insensible en la razón en sintonía respecto a $p$, para todos los periodos. En cuanto al coeficiente de pérdida de carga óptimo, aumenta en forma cuadrática respecto al aumento de razón de longitud. Además, la incertidumbre es prácticamente insensible, y para el caso de estructuras flexibles se logra apreciar una variación de la incertidumbre respecto al valor determinístico. Se observa que cuando el periodo de la estructura coincide con el periodo predominante de la excitación sísmica, $T=2 \mathrm{~s}$, el coeficiente de pérdida de carga, tiene un mayor valor.
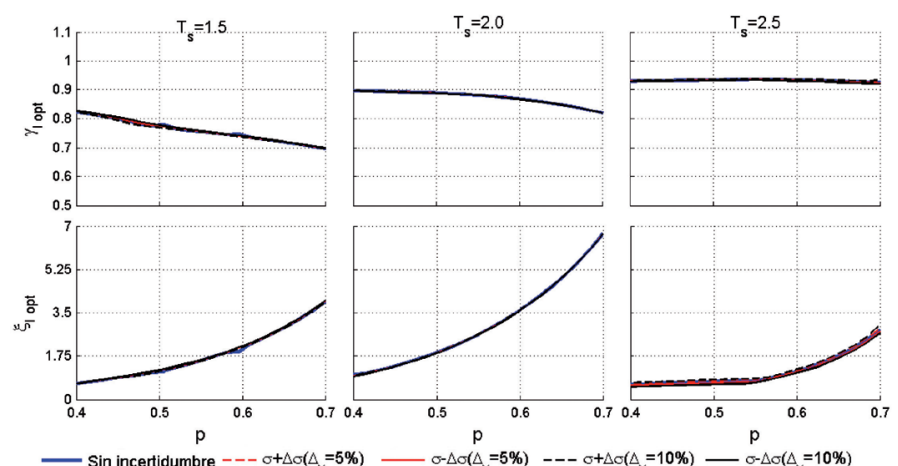

- Sin incerlidumbre $---\sigma+\Delta \sigma(\Delta \times-5 \%)$

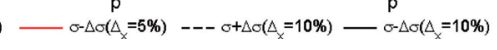

Figura 4: Parámetros óptimos del ACLS en función de la razón de longitud $p$

\section{Análisis del comportamiento de un AMS con variación de $p$}

La Figura 5 muestra que la razón de sintonía y amortiguamiento del AMS, son insensibles con respecto a la razón de longitud $p$, con respecto a la razón de sintonía óptima, para los periodos de 1.5 y $2.0 \mathrm{~s}$. Para un periodo de $2.5 \mathrm{~s}$ existe una concavidad hacia abajo y una concavidad hacia arriba, de la razón de sintonía y la razón de amortiguamiento del AMS, respectivamente. Con respecto a sensibilidad ante una incertidumbre en la razón de longitud, se observa que la razón de sintonía es levemente sensible en estructuras muy flexibles.
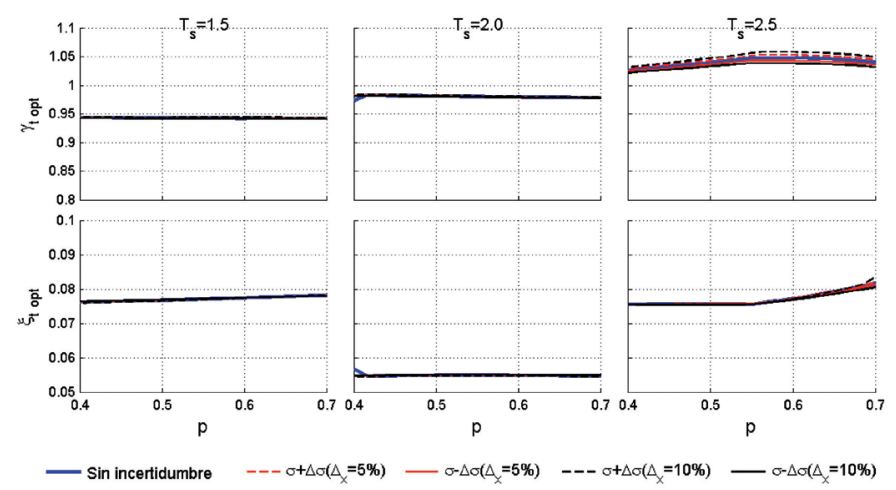

Figura 5: Parámetros óptimos del AMS en función de la razón de longitud $p$

También se observa que cuando el periodo de la estructura coincide con el periodo predominante de la excitación sísmica $\left(T_{p}=2 \mathrm{~s}\right)$, el amortiguamiento óptimo del AMS toma un valor un por debajo de las otras estructuras. 


\section{Sensibilidad de la incertidumbre en la eficiencia del ACS}

La eficiencia en la reducción del desplazamiento $\mathrm{RD}$, se define como un porcentaje de reducción de la desviación estándar de desplazamiento de la estructura principal, controlada por un ACS con parámetros óptimos, respecto a la desviación estándar de desplazamiento de la estructura sin ACS. Es decir, a mayor valor de RD, la eficiencia es mayor. Se observa en la Figura 6 que la eficiencia aumenta con respecto de la razón de masa, independiente de la flexibilidad de la estructura. Además, cuando el periodo de la estructura coincide con la frecuencia predominante del input, el ACS es más eficiente. También se observa que la eficiencia del ACS, no es sensible a incertidumbres menores a $10 \%$ en la razón de masa.

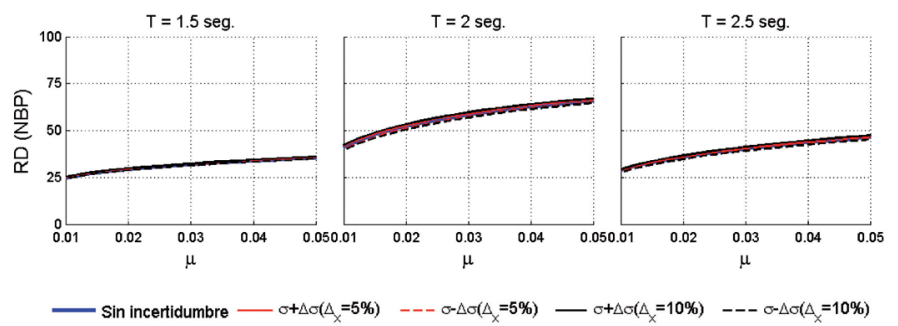

Figura 6: Eficiencia del ACS, para una incertidumbre en la razón de masa $\mu$

De la Figura 7 se observa que la eficiencia del ACS es prácticamente insensible a la razón de longitud $p$, pero sí depende del periodo de la estructura y el periodo predominante de la excitación. Esto se puede observar en el caso de una estructura de $2 \mathrm{~s}$, coincidente con el periodo predominante de la excitación. Es este caso la eficiencia es máxima. Por otra parte, se observa un leve cambio en la eficiencia del ACS, al introducir una incertidumbre de hasta un $10 \%$ en la razón de longitud.

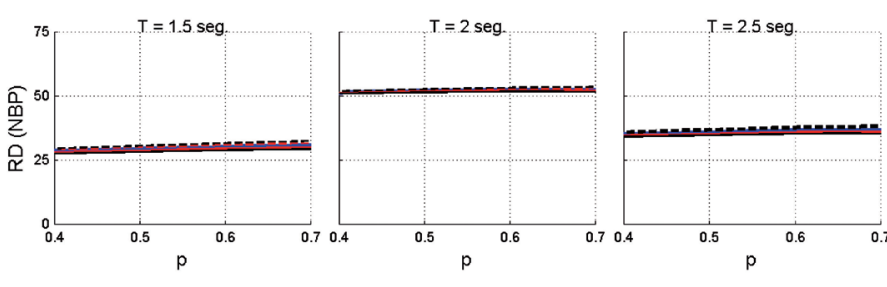

Figura 7: Eficiencia del ACS, para una incertidumbre en la razón de longitud $p$

\section{Sensibilidad de la incertidumbre en la RMS del desplazamiento ACS}

En la Figura 8 se muestra la raíz cuadrática media RMS del desplazamiento de la estructura principal, con respecto a la variación de masa, para una incertidumbre de \pm 5 y $\pm 10 \%$. Se observa que para estructuras más rígidas la RMS es insensible a la razón de masa $\mu$ y a la incertidumbre en ella. En el caso de una estructura de 2 s, se produce un aumento respecto a los otros periodos analizados debido a la resonancia que se produce con el periodo predominante del sismo. Se observa también que, para este mismo periodo, se produce un decrecimiento del RMS del desplazamiento respecto a la razón de masa. Se observa también, que existe una pequeña sensibilidad en la RMS de desplazamiento respecto a la incertidumbre de la razón de masa.

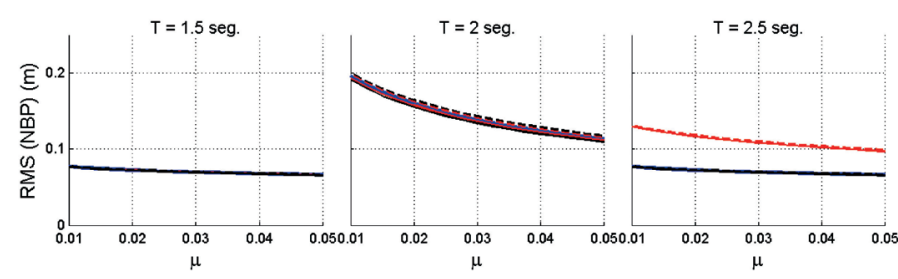

- Sin incertidumbre - $\sigma+\Delta \sigma\left(\Delta_{x}=5 \%\right) \quad--\sigma-\Delta \sigma\left(\Delta_{x}=5 \%\right)-\sigma+\Delta \sigma\left(\Delta_{x}=10 \%\right) \quad---\sigma-\Delta \sigma\left(\Delta_{x}=10 \%\right)$

Figura 8: RMS en función de la razón de masa $\mu$

Para una estructura de un periodo de $2.5 \mathrm{~s}$, se observa que para una incertidumbre de $\pm 5 \%$, se produce una amplificación importante de los desplazamientos. Lo que puede ser muy negativo en una estructura.

En la Figura 9 se muestra el RMS del desplazamiento de la estructura principal, con respecto a la variación de $p$, para una incertidumbre de $\pm 5 \mathrm{y} \pm 10 \%$. Se observa que la RMS es insensible a la razón de longitud $p$.

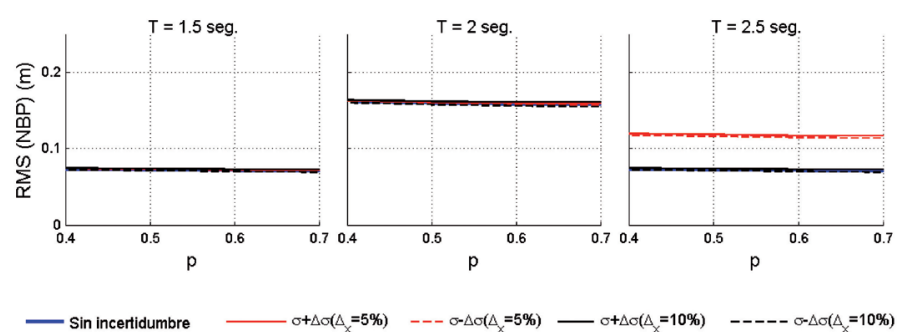

Figura 9: RMS del desplazamiento en función de la razón de longitud $p$ 
Se observa también que la RMS de desplazamiento para un periodo de $2 \mathrm{~s}$, aumenta respecto a los otros periodos, lo que se debe a la resonancia con el periodo predominante del input. Para la estructura más flexible, se observa que para una incertidumbre de $\pm 5 \%$, se produce un aumento del RMS de desplazamientos.

\section{Conclusiones}

En este estudio se analizó la respuesta de un dispositivo ACS considerando un análisis probabilístico para sismos estocásticos con una densidad de potencia espectral asociada a un bajo contenido de frecuencias. De los resultados obtenidos se puede concluir lo siguiente:

- La razón de sintonía del ACLS decrece respecto a un aumento de la razón de masa y es insensible a la incertidumbre en la razón de masa.

- El coeficiente de pérdida de carga del ACLS disminuye respecto a la razón de masa y aumenta con respecto a la razón de longitud, y en ambos casos es insensible a la incertidumbre en los parámetros.

- La razón de sintonía del AMS es insensible a un aumento de la razón de longitud.

- La eficiencia del ACS aumenta con el aumento de la razón de masa, y es mayor cuando el periodo de la estructura coincide con el periodo predominante de la excitación.

- La eficiencia del ACS es levemente sensible a la incertidumbre en la razón de masa y a la razón de longitud.

- La RMS de desplazamiento disminuye respecto a la razón de masa, no depende del valor de la razón de longitud y es mayor cuando el periodo de la estructura, coincide con el periodo predominante de la excitación.

- No se observan cambios significativos, en la eficiencia del ACS, ante una incertidumbre de un 10\% en la razón de masa o razón de longitud del ACLS.

A la luz de estos resultados, se recomienda en futuras investigaciones introducir niveles de incertidumbre mayores a $\pm 10 \%$ y también realizar análisis determinísticos para comparar con los resultados estocásticos.

\section{Agradecimientos}

Los autores agradecen a la Universidad del Bío-Bío por el apoyo dado a través del Proyecto Regular UBB 2060542 IF/R.

\section{Referencias}

Bigdeli, Y. and Kim, D. (2015). Damping effects of the passive control devices on structural vibration control: TMD, TLC and TLCD for varying total masses. KSCE Journal of Civil Engineering 20(1), 301-308

Chen, B.F. and Yang, B.H. (2018). Experimental study of a hybrid TMD and TLD on structure motion reduction. Ocean Engineering 165, 538-549

Clough, R.W. and Penzien, J. (1975). Dynamics of structures. McGraw-Hill, USA

Debbarma, R., Chakraborty, S. and Ghosh, S. (2010). Unconditional reliability-based design of tuned liquid column dampers under stochastic earthquake load considering system parameters uncertainties. Journal of Earthquake Engineering 14(7), 970-988

Di Matteo, A., Lo Iacono, F.L., Navarra, G. and Pirrotta, A. (2015). Innovative modeling of tuned liquid column damper motion. Communications in Nonlinear Science and Numerical Simulation 23(1-3), 229-244

Di Matteo, A., Pirrotta, A. and Tumminelli, S. (2017). Combining TMD and TLCD: analytical and experimental studies. Journal of Wind Engineering and Industrial Aerodynamics 167, 101-113

Espinoza, G., Sagredo, G. y Suazo, A. (2020). Análisis de la eficiencia de un amortiguador combinado sintonizado con incertidumbre en los parámetros sometido a una excitación sísmica de alto contenido de frecuencias. Obras y Proyectos $\mathbf{2 8}$, $58-67$

Espinoza, G., Carrillo C. and Suazo, A. (2018a). Analysis of a tuned liquid column damper in non-linear structures subjected to seismic excitations. Latin American Journal of Solids and Structures 15(7), e91

Espinoza, G., Benedetti, F., Alvarez-Mendoza, P. and Bonilla, E. (2018b). Influence of the seismic excitation frequencies content on the behavior of a tuned mass damper in low-rise building considering soil-structure interaction. Latin American Journal of Solids and Structures 15(8), e75 
Lyapunov, A.M. (1992). The general problem of the stability of motion. Taylor \& Francis, UK

Saitua, F., Lopez-Garcia, D. and Taflanidis, A.A. (2018). Optimization of height-wise damper distributions considering practical design issues. Engineering Structures 173, 768-786

Sakai, F. (1989). Tuned liquid column damper-new type device for suppression of building vibration. First International Conference on High-Rise Buildings, Nanjing, China, 926-931

Schmelzer, B., Oberguggenberger, M. and Adam, C. (2010). Efficiency of tuned mass dampers with uncertain parameters on the performance of structures under stochastic excitation. Proceedings of the Institution of Mechanical Engineers, Part $O$ : Journal of Risk and Reliability 224(4), 297-308
Shum, K.M. (2009). Closed form optimal solution of a tuned liquid column damper for suppressing harmonic vibration of structures. Engineering Structures 31(1), 84-92

Tributsch, A. and Adam, C. (2012). Evaluation and analytical approximation of Tuned Mass Damper performance in an earthquake environment. Smart Structures and Systems 10(2), 155-179

Wang, L., Zhao, X. and Zheng, Y.M. (2016). A combined tuned damper and an optimal design method for wind-induced vibration control for super tall buildings. The Structural Design of Tall and Special Buildings 25(10), 468-502 